\title{
Acoustooptic digital filter
}

\author{
Fariborz Salamat and Robert J. Marks II
}

\begin{abstract}
Digital filters have three components: (1) unit delays, (2) multipliers, and (3) adders. These components can be implemented by using (1) a Bragg cell as a tapped delay line, (2) optical multiplication using the Bragg cell transmittance, and (3) photodetectors. Multiplexing techniques are shown to increase geometrically the data handling capacity of such filters. An architecture for a 2-D digital filter using such techniques is presented.
\end{abstract}

\section{Introduction}

Acoustooptic cells have been used extensively in optical computing for the operations of matrix multiplications and convolution-type operations. ${ }^{1-7}$ Some digital convolutions can be performed by digital filters ${ }^{8,9}$ with the advantage of no required finite windowing.

Digital filters consist of three basic components: unit delays; multipliers; and adders. Unit delay operation can be performed by a Bragg cell identical in concept to a tapped transmission line implementation of a unit delay. ${ }^{9}$ Multipliers can be implemented by conventional optical multiplication using the Bragg cell transmittance. Addition can be performed by photodetectors.

Using the above elements, we propose a basic architecture for both finite and infinite impulse response (FIR and IIR) digital filters. The use of multiplexing is addressed, and the filter's potential is shown to be in billions of operations per second (BOPS). Using multiplexing techniques, an architecture for 2-D digital filtering is presented.

\section{Preliminaries}

\section{A. Digital Filter} by

A general expression for a 2-D digital filter is given

$$
y(n)=\sum_{i=0}^{I} a_{i} v(n-i)+\sum_{j=1}^{J} b_{j} y(n-j),
$$

where $v(n)$ is the input, $y(n)$ is the output, and $a_{i}$ and

The authors are with University of Washington, Department of Electrical Engineering, Seattle, Washington 98195.

Received 20 August 1984.

0003-6935/85/060829-07\$02.00/0.

(C) 1985 Optical Society of America. $b_{j}$ are weighting coefficients. From this expression it can be seen that the filter is composed of the following components: (1) delay elements; (2) multipliers; and (3) adders. Therefore, any system that proposes to implement a digital filter must contain these three elements. Acoustooptic interactions provide us with first two of these elements with the third implemented by photodetectors in simpler cases. In more complicated applications, summation together with bookkeeping functions is left to the electronics of the system.

\section{B. Acoustooptic Interaction}

It has been shown that weak acoustooptic interaction in the Bragg regime can be used to multiply numbers. ${ }^{10}$ Here we present a summary of this interaction (for a detailed discussion see Rhodes ${ }^{2}$ ).

Figure 1 shows an acoustooptic (AO) medium where an acoustic wave with velocity $V$ and frequency $f_{0}$ propagates. This wave generates a strain wave in the AO medium of the form

$$
s(x-V t)=c(x-V t) \cos \left[2 \pi \dot{f}_{0}(x-V T)+\alpha(x-V t)\right],
$$

where $c(x, t)$ and $\alpha(x, t)$ are the amplitude and phase of the wave, respectively. $x$ is the direction of the propagation of the acoustic wave, $t$ denotes time, and $x$ and $t$ are related by the velocity of the acoustic wave. If the amplitude $c(x, t)$ is sufficiently small, the effective transmittance of the $\mathrm{AO}$ medium in the Bragg regime of operation is given by

$$
t(x, t)=\left\{1+j 1 / 2 c(x-V t) \exp [j \alpha(x-V t)] \exp \left[j 2 \pi f_{0}(x-V t)\right]\right\} .
$$

Now if the medium is addressed by an optical wave of frequency $\nu$ and amplitude $\sqrt{a}$, the resulting transmitted wave $u(x, t)$ will be

$$
u(x, t)=\left\{1+j^{1} / 2 c(x-V t) \exp \left[j 2 \pi f_{0}(x-V t)\right] \sqrt{a} \exp (j 2 \pi \nu t)\right\},
$$

where we have set $\alpha(x-V t)=0$ for convenience. The corresponding intensity of the output will be

$$
I=|u(x, t)|^{2}=a+1 / 4 c^{2} a-a c \sin 2 \pi f_{0}(x-V t) .
$$




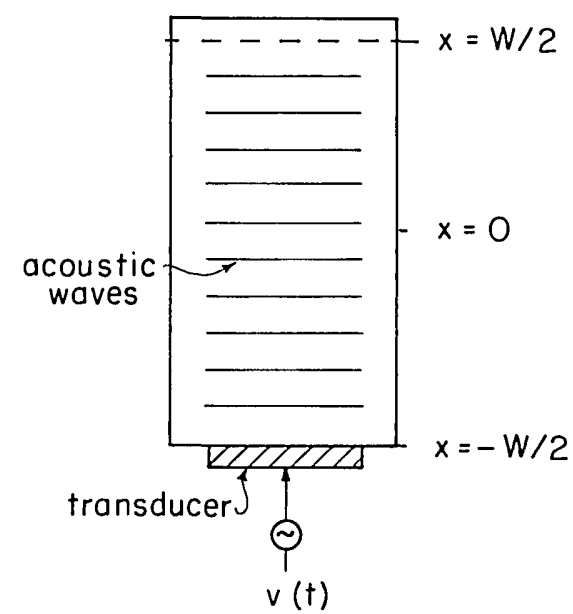

Fig. 1. Acoustooptic medium: Acoustic waves are generated by the acoustic transducer which is fed by the electrical signal $v(t)$. The acoustic wave is absorbed at $x=w / 2$ to avoid reflections. Length of the medium is $w$.

It can be seen that the last term is the product of the acoustic strain wave and the square of the optical amplitude to within a phase difference. This term can be extracted by bandpass/highpass filtering the intensity, the other two terms having their frequency components far removed.

Another feature of the AO medium is that it behaves as a series of delay elements. That is, it not only contains the current input signal but the previous inputs up to $W / V$ sec before, $W$ being the length of the medium (see Fig. 1).

As we will see in the next section, the AO interaction and $\mathrm{AO}$ medium provide us with the multipliers and the delay elements of the digital filter.

\section{Basic System Architecture}

Suppose an electrical signal $u(t)$ is to be filtered. It first has to be preprocessed as shown in Fig. 2. A sample and hold operation on the input signal $u(t)$ gives $u[n]=u(n T)$, with $T$ the sampling period. Our acoustic medium is geneally operable in a given frequency range, so it is also necessary to put the sampled signal on a carrier frequency $f_{0}$ in that range. Then the electrical signal fed to the acoustic transducer will be given by

$$
v(t)=c[n] \cos \left(2 \pi f_{0} n T+\alpha[n]\right) ; \quad n T<t<(n+I) T,
$$

where $c[n]$ and $\alpha[n]$ are the amplitude and phase of $u[n]$, respectively.

At this point, for ease of presentation, let us assume that $\alpha[n]=0$; then $v(t)$ is a continuous signal of frequency $f_{0}$ with amplitude $c[n]$ lasting for one sampling period $T$. For the next $T \sec v(t)$ will have an amplitude $c[n+1]$ and so on.

Now if $v(t)$ is fed to an acoustic transducer which converts the electrical signal $v(t)$ to mechanical vibrations, acoustic waves will be launched in the acoustooptic medium.

If the speed of the acoustic wave in the acoustooptic medium is given as $V$, the signal appearing at $x_{1}$ is the

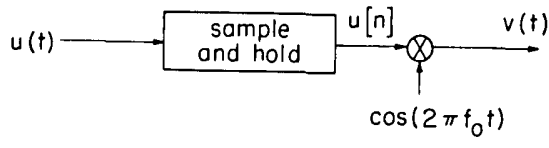

Fig. 2. Preprocessing of the input signal.

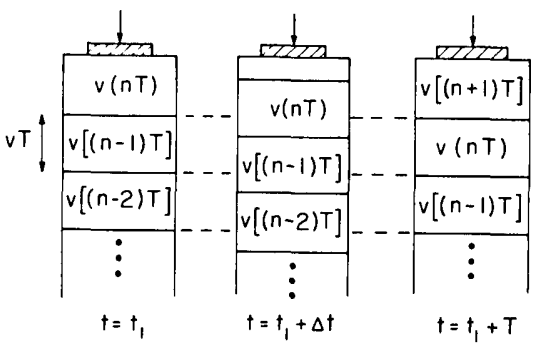

Fig. 3. Snapshots of the propagation of the signal in $\mathrm{AO}$ medium at $t=t_{1}, t_{1}<t<t_{1}+T$, and $t=t_{1}+T$. The length of each cell is $V T$.

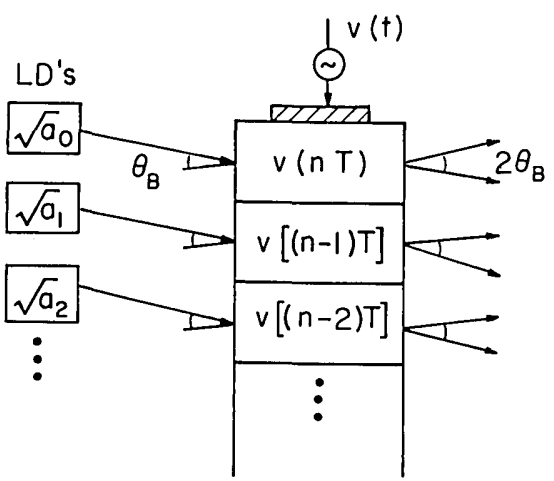

Fig. 4. AO medium addressed by LDs of amplitudes: $\sqrt{a_{0}}$, $\sqrt{a_{1}}, \ldots$

signal that was at $x_{1}-V t_{1}, t_{1}$ sec before. Therefore, if we divide the $\mathrm{AO}$ medium into cells each of length $V T$, we will have the configuration shown in Fig. 3. (Similar techniques have been used elsewhere; for example, see Ref. 5.)

Figure 3 shows the progress of the acoustic signal with time in the AO medium. The signal $v(n, t)$ which is in the first cell at time $t_{1}$ will be in the second cell after $T$ sec (length of each cell being $V T$ ), and for time $t_{1}<t<$ $t_{1}+T$ it is partially in cell one and partially in cell two. There is an attenuation of the signal as it travels through the medium, which we will ignore since it can be compensated for easily. Also, the medium is of finite length so we have a limited number of cells to work with.

Now let us position a number of laser diodes (LDs) so that each of them addresses one of the imaginary cells at the Bragg angle $\theta_{\mathrm{B}}$ (see Fig. 4). Considering the $i$ th cell with its corresponding $L D$ of amplitude $\sqrt{a_{i}}$, we can see that, as discussed before, we get a product term of $a_{i} v[n-i]$, where $v[n-i]$ is the acoustic signal in the $i$ th cell. $v[n-i]$ is $v(t)$ when $(n-i) T<t<(n-i+1) T$. An isolated cell is shown in Fig. 5. In this figure the output of the photodetector (PD) is the intensity $I(x, t)$, 


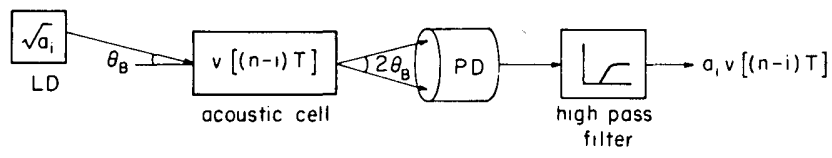

Fig. 5. One-cell operation.

and, as mentioned before, this is bandpass/highpass filtered to give the product term.

At this point it must be obvious that this system can be used as a digital FIR filter

$$
y[n]=a_{0} v[n]+a_{1} v[n-1]+a_{2} v[n-2]+\ldots,
$$

where $v[n]$ is the input to the system, $y(n)$ is the output, and each product term corresponds to a given cell and its LD.

Once we have the product terms, they can be summed to give $y[n]$. An IIR filter follows naturally, as shown in Fig. 6. Note that only one PD is needed since the LDs are mutually incoherent and no cross terms appear in the calculation of the intensity. In this scheme, the PD not only generates the intensity terms for each cell but also adds the product terms at the same time. In more complicated cases, however, we might need one PD for each cell since it might be necessary to add the product terms selectively, and the one PD technique will no longer be sufficient. Of course, if the LDs are in any way correlated the one $\mathrm{PD}$ technique can no longer be used.

\section{A. Filter Coefficients}

Suppose that we want to implement the following filter:

$$
\begin{aligned}
y[n]= & a_{0} v[n]+a_{1} v[n-1]+a_{2} v[n-2]+\ldots \\
& +b_{1} y[n-1]+b_{2} y[n-2]+\ldots
\end{aligned}
$$

Assuming the filter to be shift-invariant we can implement the coefficients with LDs together with appropriate neutral density filters. If our LDs are all identical, a LD without a neutral density filter corresponds to the maximum amplitude available for a coefficient. Therefore, if our maximum coefficient is $a_{p}$, we write

$$
\begin{aligned}
y[n]= & a_{p}\left\{a_{0}^{\prime} v[n]+a_{1}^{\prime} v[n-1]+\ldots\right. \\
& \left.+b_{1}^{\prime} y[n-1]+b_{2}^{\prime} y[n-2]+\ldots\right\},
\end{aligned}
$$

where $a_{i}^{\prime}=a_{i} / a_{p}$ and $b_{j}^{\prime}=b_{j} / a_{p}$, and the $a_{p}$ multiplier can be easily realized in the electronics after the summer.

The second problem we might have with the coefficients is that they might be negative. There are several techniques that enable us to handle negative coefficients (see, for example, Ref. 11). Two of these are considered here:

(a) We can have two summers, one for the positive and the other for the negative coefficients. In this case, the output of the PD (each cell has its own PD) can be switched to either of two summers. If the coefficient for the cell is negative, the PD corresponding to it is switched to the summer for negative values and vice versa. This is done for all PDs, and then the output of the two summers is subtracted.

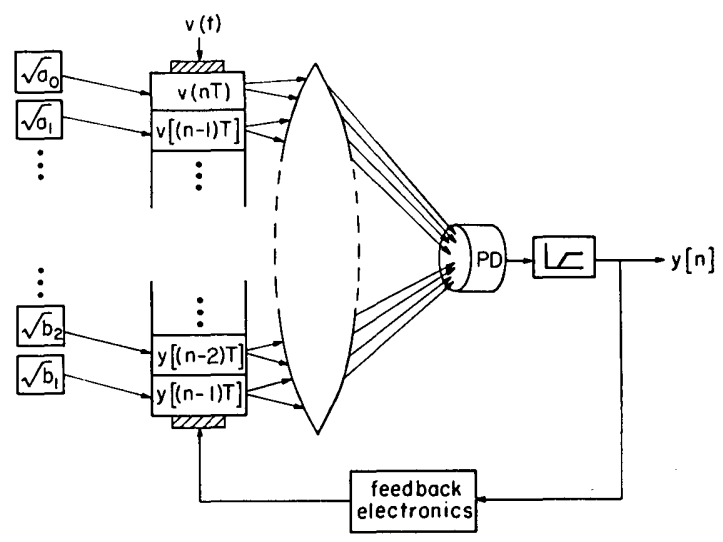

Fig. 6. IIR filter: Output of the bandpass filter is $y[n]$, which is placed on a carrier frequency and fed back to another $\mathrm{AO}$ medium to generate $b_{j} y[n-j]$ terms.

(b) We may have two time intervals in each period $T$. In this method, we collect two sets of data; i.e., we sum twice. During one interval we disable, say, the negative coefficients; that is, we turn off the LDs corresponding to negative values so we get the equivalent of positive output of method (a); then we disable the positive $L D s$ and get the negative output and then we subtract the two. Note that while in method (a) we have abandoned the one photodetector technique; in method (b) we can still use it.

\section{Multiplexing}

One of the best attributes of the acoustooptic digital filters is the dimensions available for multiplexing. These dimensions are (1) time, (2) space, (3) acoustic frequency, (4) optical frequency, (5) angle, and (6) polarization.

\section{(1) Time}

By time we mean any changes in the system in real time. This includes, for example, changes in the filter coefficients for a shift-variant filter or a system that can realize several filters and hence requires a change of the coefficients. With time we could also change the manner in which the electronics of the system operates. For example, recall the one-summer handling of the negative coefficients.

\section{(2) Space}

For this dimension we just mention that we can have a 2-D array instead of the 1-D one we have been considering and that a given cell may be addressed by several LDs.

\section{(3) Acoustic Frequency}

Each AO medium has a given bandwidth, so we can feed it with several, instead of one, acoustic frequencies within that bandwidth. Since the Bragg angle is given by $\theta_{\mathrm{B}}=k_{a} / 2 K=\lambda / 2 \Lambda$, where $\lambda$ is the optical wavelength and $\Lambda$ is the acoustical wavelength, we see that as $\Lambda$ changes so does the Bragg angle, and, therefore, each acoustic frequency generates a diffracted beam which carries the information as covered before (see Fig. 


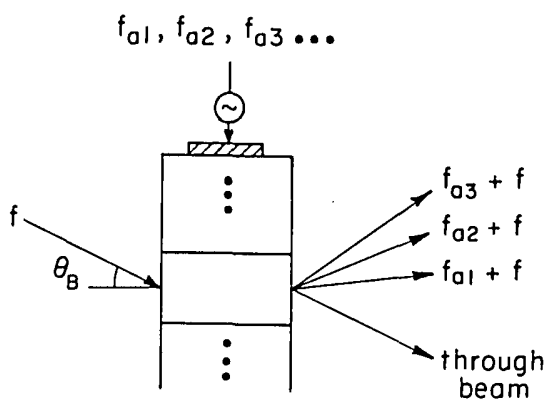

Fig. 7. Acoustic frequency multiplexing.

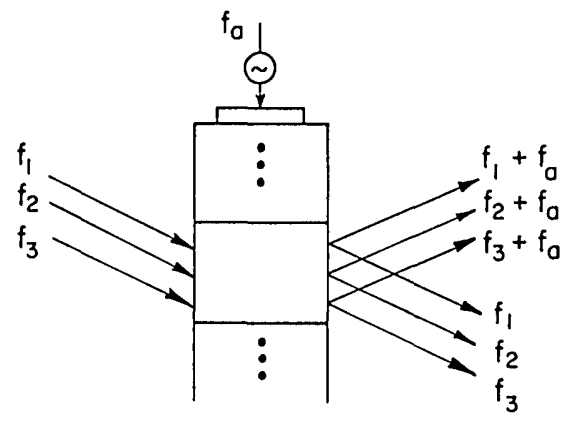

Fig. 8. Optical frequency multiplexing.

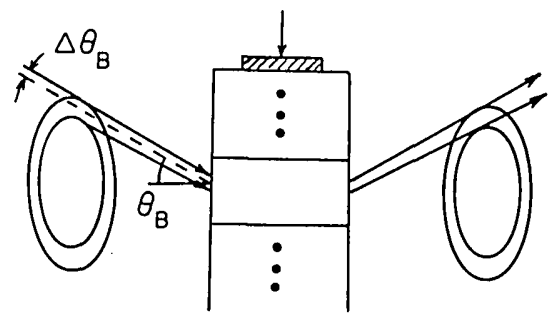

Fig. 9. Angular multiplexing.

7). This makes it possible to filter several signals (Each on a different acoustic frequency) at the same time.

The reader might note that since $\theta_{\mathrm{B}}$ is a function of the acoustic frequency, just one of the acoustic frequencies is addressed at its exact Bragg angle. However, we maintain that there is a range of angles $\theta_{\mathrm{B}}$ $\pm \Delta \theta_{\mathrm{B}}$ that would still yield useful results. Also, we could address the cell at different angles by using several LDs [see (2) and (5)].

\section{(4) Optical Frequency}

This is quite similar to the acoustic multiplexing except that we change the optical frequency and again get different diffracted beams for each optical frequency (see Fig. 8). This means that several distinct filtering operations can be done on the same signal, since each optical frequency can correspond to a set of coefficients and hence different filtering operations.

Acoustooptic Frequency Multiplexing. The two frequency multiplexing techniques, $n$ acoustic frequencies and $m$ optical frequencies, can be combined to result in $\mathrm{nm}$ useful diffracted components, which may be employed to realize a wide variety of systems the user might want to implement. This is a tremendous capability, as well be demonstrated in the realization of 2-D filtering.

\section{(5) Angular Multiplexing}

Yet another dimension of the system is realized by noting that the Bragg angle $\theta_{\mathrm{B}}$ in fact corresponds to a cone, see Ref. 12. That is, a cell can be addressed at any angle $\alpha$ which satisifies $\alpha=\theta_{\mathrm{B}} \pm \Delta \theta_{\mathrm{B}}$, where $\Delta \theta_{\mathrm{B}}$ is the angular increment that still yields useful results although the incidence angle is not exactly $\theta_{\mathrm{B}}$ (see Fig. 9).

With this constraint we get a cone to address the cell, and this in turn generates a cone of diffracted beams where each beam can carry a distinct information.

\section{(6) Polarization}

As the last dimension, we mention multiplexing by polarizing the optical wave. We can distinquish two diffracted beams by their polarization, and hence we can address the cell by two mutually perpendicularly polarized optical beams where they both could have the same optical and acoustical frequencies and still be distinquishable.

\section{System Capability}

The limiting factor in the speed of the system is the restriction on $c(x-V t)$ in Eq. (1) to be of lower frequency than the acoustic frequency $f_{0}$ so that we could use a bandpass filter to extract the product term. To illustrate the speed of the system, we note that acoustic frequency is generally between $1 \mathrm{MHz}$ and $1 \mathrm{GHz}$, so for purposes of illustration let it be $50 \mathrm{MHz}$. Then frequency of $c(x-V t)$ will be $\sim 5 \mathrm{MHz}$, which implies a sampling period of $200 \mathrm{nsec}$. Note that after each pe$\operatorname{riod} T$ we get an output so we have a device speed of 5 million operations or outputs per second (MOPS).

In support of this estimate, consider the following:

(1) The cell length will be of the order $V T=1 \mathrm{~cm}$, where we have used a typical velocity. This is quite large, and, therefore, we have no problem accommodating the $\mathrm{LD}$ and $\mathrm{PD}$ or other system components.

(2) It might be possible to use higher acoustical frequencies, in which case the frequency of $c(x-V t)$ can be higher too, and hence more MOPS will be realizable.

(3) The phase-to-amplitude optics, specifically, the lens shown in Fig. 10, can be realized by a fly's eye (or a phase hologram) and can be quite small, so there is no serious problem in having smaller cells as far as the optics of the device is concerned.

(4) In the manufacturing phase of production of the system, it might be possible to have a much higher density.

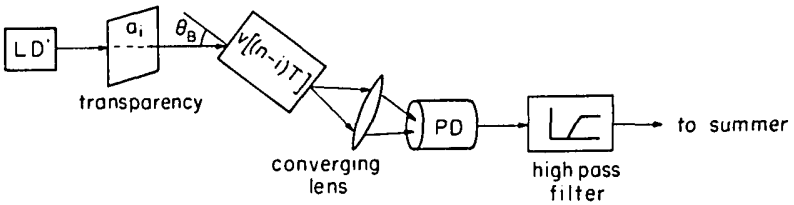

Fig. 10. Detailed illustration of a one-cell operation. 
(5) In addition, we have the dimensionality of the system. Let us assume that time, space, angular dimension, and polarization each doubles the MOPS. Frequency multiplexing composed of optical and acoustic multiplexing has a great potential, and let us suppose that each of these just quadruples the number of operations. This gives 1.28 BOPS, which is a staggering number of operations, exceeding one billion operations per second, which is 3-4 orders of magnitude greater than the fastest digital filter implemented by the computers.

As the above arguments suggest, the 5 MOPS are to be considered a conservative estimate, and the MOPS could go higher after multiplexing and using a tailormade system.

\section{Two-Dimensional Filtering}

A 2-D filter is given by the following expression:

$$
\sum_{p} \sum_{q} b[p, q] y[n-p, m-q]=\sum_{k} \sum_{i} a[k, i] v[n-k, m-i] .
$$

For the following presentation, we assume $a$ and $b$ coefficients to be constant, although they need not be so, as discussed for the 1-D case, but it simplifies the presentation. Also, since the treatment of the IIR case is an extension of the FIR case, we treat the following 2-D filter instead:

$$
y[n, m]=\sum_{k=0}^{K} \sum_{i=0}^{I} a[k, i] v[n-k, m-i] .
$$

To understand how the system accommodates this filter, we start with an illuminating example and then present the general case.

Suppose $k$ and $i$ solely take the values zero and one; then the $y[n, m]$ outputs will be given as follows:

$$
\text { (a) } \begin{aligned}
y[0,0] & =a[0,0] v[0,0], \\
y[0,1] & =a[0,0] v[0,1]+a[0,1] v[0,0], \\
y[0,2]= & a[0,1] v[0,1] ; \\
\text { (b) } y[1,0]= & a[0,0] v[1,0]+a[1,0] v[0,0], \\
y[1,1]= & a[0,0] v[1,1]+a[0,1] v[1,0] \\
& +a[1,0] v[0,1]+a[1,1] v[0,0], \\
y[1,2]= & a[0,1] v[1,1]+a[1,1] v[0,1] ; \\
\text { (c) } y[2,0]= & a[1,0] v[1,0], \\
y[2,1]= & a[1,0] v[1,1]+a[1,1] v[1,0], \\
y[2,2]= & a[1,1] v[1,1] .
\end{aligned}
$$

The reason we have grouped these outputs will become clear as we proceed.

Let us consider Fig. 11, which generates outputs for part (a). It can be seen that as $v[0,0]$ comes into cell one and is multiplied by $a[0,0]$, we get $y[0,0]$. Next, as $v[0,0]$ travels to cell two and $v[0,1]$ travels to cell one, we get $y[0,1]$ by adding the resulting products. Next, as $x[0,1]$ travels to cell two, we get $y[0,2]$. Note that we do not get terms like $a[0,0] v[0,2]$ because of our assumption on $k$ and $i$.

Similarly, part (b) is generated by the arrangement in Fig. 12. To generate part (c) outputs, we propose a

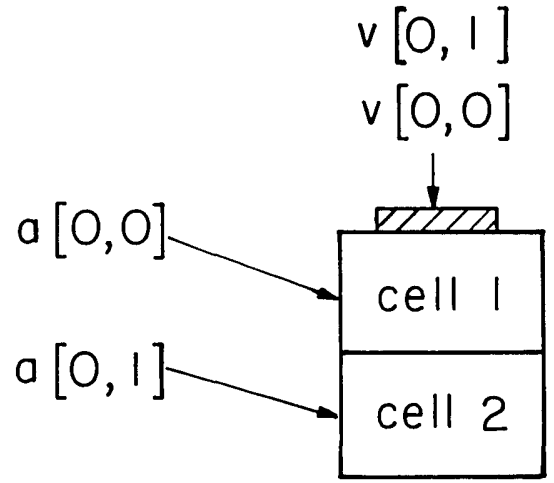

Fig. 11. Generation of part (a) outputs. (The square root factor is not shown for clarity.)

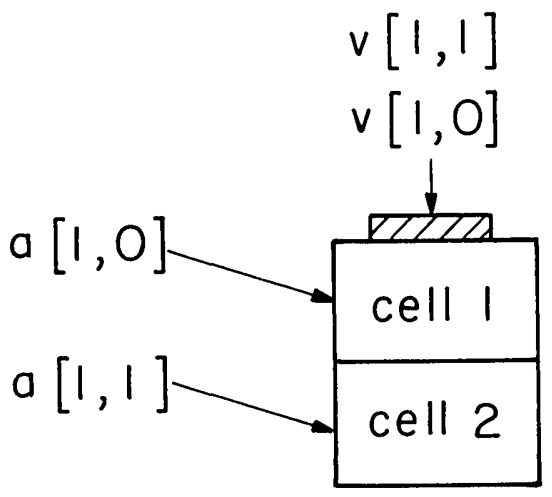

Fig. 12. Generation of part (b) outputs.
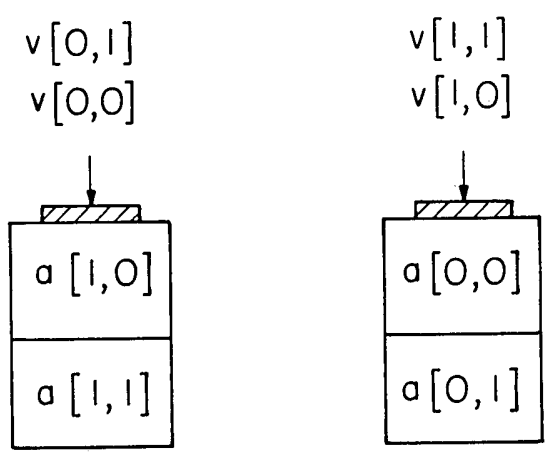

Fig. 13. Generation of part (c) outputs.

simple extension of the system as shown in Fig. 13, where for purposes of illustration the coefficients are placed over the cell they address. The system works as follows: First $v[0,0]$ and $v[1,0]$ travel to first cells of their respective mediums and are multiplied by the coefficients addressing them, yielding $y[1,0]$ as they are added. After one period elapses, $v[0,0]$ and $v[1,0]$ are now in cells marked two, and $v[0,1]$ and $v[1,1]$ are in cells marked one, and we get $y[1,1]$, and after another period $T$ we get $y[1,2]$.

Together the above schemes give us all the outputs. But note that the last scheme could also give us all the outputs if we use acoustical frequency multiplexing, as shown in Fig. 14. Specifically, 


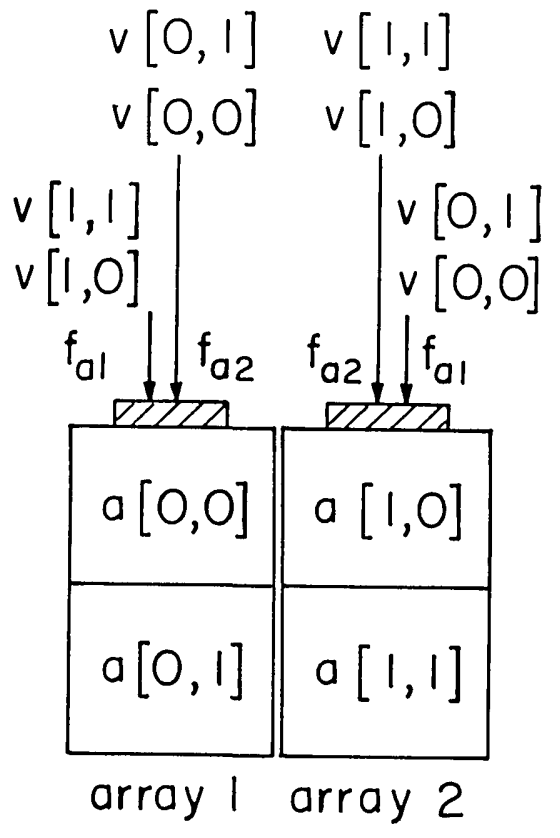

Fig. 14. Acoustical multiplexing for a simple 2-D filter.

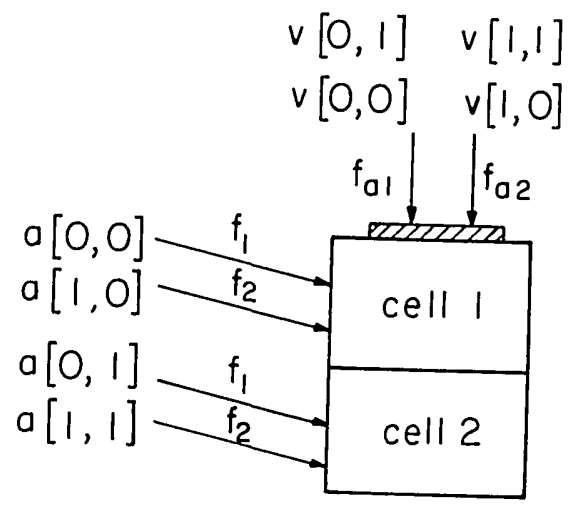

Fig. 15. Simple 2-D filter.

$f_{a 2}$ and array 1 are equivalent to Fig. 11 ,

$f_{a 2}$ and array 2 are equivalent to Fig. 12, and

$f_{a 1}$ and both arrays are equivalent to Fig. 13.

Now we take this one step further, and by using optical frequency multiplexing we attempt to use just one array. This is illustrated in Fig. 15.

It can be seen that

$\left(f_{a 1}, f_{1}\right)$ is equivalent to Fig. 11 ,

$\left(f_{a 2}, f_{2}\right)$ is equivalent to Fig. 12 , and

$\left(f_{a 1}, f_{2}\right)$ and $\left(f_{a 2}, f_{1}\right)$ are equivalent to Fig. 13.

Now that we have seen how the system accommodates 2-D filtering for a simple case, we treat the general case

$$
y[n, m]=\sum_{k=0}^{N_{1}-1} \sum_{i=0}^{N_{2}-1} a[k, i] v[n-k, m-i] .
$$

Let $v[i, j]$ be given by the acoustic frequency for $i$ and time for $j$ as in Table $\mathrm{I}$, and let $a[i, j]$ be given by the optical frequency for $i$ and $x$ dimension for $j$ as in Table II. Then, as is illustrated in Fig. 16,
Table I. Inputs of the Two-Dimensional Filter

\begin{tabular}{|c|cccc|}
\hline & $f_{a}^{0}$ & $f_{a}^{1}$ & $\cdots$ & $f_{a}^{N_{1}-1}$ \\
\hline$t=0$ & $v[0,0]$ & $v[1,0]$ & $\cdots$ & $v\left[N_{1}-1,0\right]$ \\
$t=1$ & $v[0,1]$ & $v[1,1]$ & $\cdots$ & $v\left[N_{1}-1,1\right]$ \\
$\dot{t}$ & $\dot{0}$ & $\dot{v}$ & & $\vdots$ \\
\hline$N_{2}-1$ & $v\left[0, N_{2}-1\right]$ & $v\left[1, N_{2}-1\right]$ & $\cdots$ & $v\left[N_{1}-1, N_{2}-1\right]$ \\
\hline
\end{tabular}

Table II. Coefficients of the Two-Dimensional Filter

\begin{tabular}{|c|cccc|}
\hline & $f^{0}$ & $f^{1}$ & $\cdots$ & $f^{N_{1}-1}$ \\
\hline$x=0$ & $a[0,0]$ & $a[1,0]$ & $\cdots$ & $a\left[N_{1}-1,0\right]$ \\
$x=1$ & $a[0,1]$ & $a[1,1]$ & $\cdots$ & $a\left[N_{1}-1,1\right]$ \\
$\dot{x}$ & $\cdot$ & $\vdots$ & & $\vdots$ \\
\hline & $\vdots$ & $\vdots$ & & $\vdots$ \\
\hline
\end{tabular}

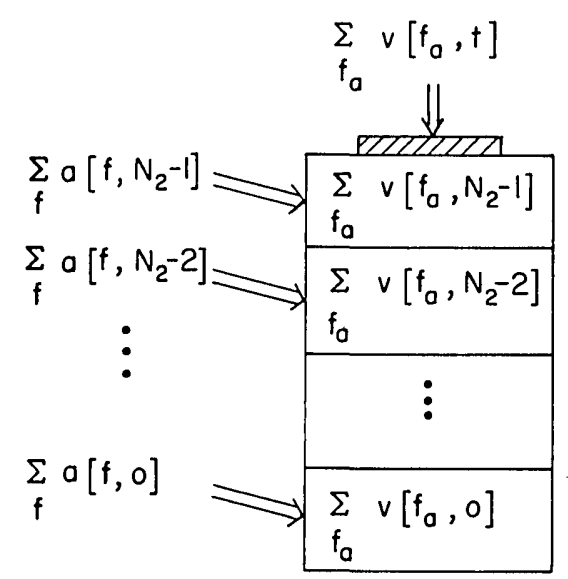

Fig. 16. General 2-D filter.

$$
y[n-m]=\sum_{k=0}^{N_{1}-1} \sum_{i=0}^{N_{2}-1} a\left[f^{k}, x^{i}\right] v\left[f_{a}^{n-k}, t^{m-i}\right],
$$

where $x^{i}$ refers to position $x=i, t^{m-i}$ to time $t=m-$ $i$, and $f_{a}^{n-k}$ and $f^{k}$ are as given in Tables I and II.

Note that the output $y[n, m]$ is generated in the following order: $y[n, 0], y[n, 1], \ldots, y\left[n, N_{2}-1\right]$ with $n=$ $0,1, \ldots, N_{1}-1$, which is the order of inputing $y[n, m]$ in the IIR case. So all the variables necessary will be available for IIR implementation of the filter.

It can be seen that the general 2-D filtering becomes very involved as $N_{1}$ and $N_{2}$ become large; yet it does so in an orderly fashion, and it is tractable. Also most of the additions and which terms are to be added together are left to the electronics of the system.

It must be mentioned that acoustical and optical frequency multiplexing are not the only dimensions 
available for implementing the 2-D digital filter. We could as well use several Bragg cells (space dimension), each accommodating a portion of the acoustical frequency multiplexing necessary. In addition, we could use time dimensions, i.e., during the period $\Delta t<T$ that the $v[n-i]$ can be addressed in cell $i$, we could divide $\Delta t$ to several smaller time intervals; during each we enable only a portion of the optical frequencies.

Therefore, we can see that although 2-D digital filtering is very involved, the dimensions of the system are a powerful tool for realizing this filter.

\section{Conclusion}

This paper introduced a new technique for implementing digital filters. We wanted a system that would be faster than its computer counterpart and have suggested an optic-acoustic-electronic hybrid device which combined the inherent characteristics of each of these fields into a system for implementing digital filters.

We have shown that the system is capable of 1 - and 2-D filtering or mathematically equivalent operations like solving differential equations or implementing convolution without the windowing effect. These operations can be FIR or IIR, shift-variant, or shift-invariant. Several filtering operations can be done on a signal, or the same filtering operation can be done on several signals. For the general case, several filtering operations can be done on several signals.

The enormous capability of the system using its dimensionality and one of its applications, 2-D filtering, was discussed.

\section{References}

1. H. J. Caulfield, J. A. Neff, and W. T. Rhodes, "Optical Computing: The Coming Revolution," Laser Focus 19, 100 (1983).

2. W. T. Rhodes, "Acousto-Optic Signal Processing: Convolution and Correlation," Proc. IEEE 69, 65 (1981).

3. T. M. Turpin, "Spectrum Analysis Using Optical Processing," Proc. IEEE 69, 79 (1981).

4. D. Casasent, "Acousto-Optic Linear Algebra Processors: Architectures, Algorithms and Applications," Proc. IEEE 72, 831 (1984).

5. D. Casasent, J. Jackson, and C. Neuman, "Frequency Multiplexed and Pipelined Iterative Optical Systolic Array Processors," Appl. Opt. 22, 115 (1983).

6. W. T. Rhodes and P. S. Guilfoyle, "Acousto-Optic Algebraic Processing Architectures," Proc. IEEE 72, 820 (1984).

7. F. Salamat, "Acousto-Optic Digital Filtering," Thesis, U. Washington (June 1984).

8. A. V. Oppenheim and R. W. Schafer, Digital Signal Processing (Prentice-Hall, Englewood Cliffs, N.J., 1975).

9. A. Papoulis, Circuits and Systems (Holt, Rinehart, \& Winston, New York, 1980.

10. Acousto-Optic Signal Processing Theory and Implementation, N. J. Berg and J. N. Lee Eds. (Marcel Dekker, New York, 1983).

11. D. E. Dudgeon and R. M. Mersereau, Multidimensional Digital Signal Processing (Prentice-Hall, Englewood Cliffs, N.J., 1984).

12. R. J. Collier, C. B. Burckhardt, and L. H. Lin, Optical Holography (Academic, New York, 1971).
We were happy to see work on the nascent question of the relationship between the nonlinear phenomena of chaos and solitons. Two papers tell us that soliton interactions and soliton imperfections give important contributions in models of the transition to chäos. Another of our questions addressed was whether the canonical fluid equations for Taylor-Couette flow are truly consistent with observed chaotic behavior. In contrast to Rayleigh-Bénard convection, however, the subharmonic bifurcations or lack thereof seem to still be a theoretical puzzle. Other articles in the general area of fluid dynamics are on methods for determining scaling exponents in vortex stretching, the role of computer graphics in 2-D flows, scalar magnetohydrodynamic models, the Painlevé criterion for integrability/inverse scattering theory, and the dominance of nonlinearity for low viscosity (small Prandtl number).

It should be emphasized that this Springer tract is largely numerical/analytical theorizing with limited experimental discussion. How well the ideas are doing and how broadly they can be applied to turbulence are problematic, as is the question of whether any proceedings reports fast-moving or fast-ending progress. In addition to the confined flows already mentioned we do find detailed success for the 1-D map model of a complex chemical reaction. Also, there is the possibility that higher degrees of freedom can be studied and may be successfully modeled in optical turbulence; this is important in our quest for a statistical mechanics of dynamical systems. Further nourishment will come, no doubt. For example, recent news about volcanic solitons may lead to another tract on geophysical applications of these exciting ideas.

ROBERT W. BROWN

Transmission Electron Microscopy. By L. REIMER. Springer-Verlag, New York, 1984. 521 pp. $\$ 46.00$.

This is an excellent book. The explosive growth of the field of transmission electron microscopy in recent decades has resulted from the extraordinary versatility of the instrument and now means that any book on the subject must cover a vast range of scientific disciplines, from microbiology, materials science, channeling, and electron optics to scattering theory, crystallography, spectroscopy, and radiation damage. To chart a course through such an ocean of material for a book of limited size is a treacherous task requiring a brave spirit; it is almost certain to overlook entire subdisciplines and offend many through neglect. Yet the writer of a text such as this, which provides a unified coherent treatment of the subject and which is pedagogically sound, performs an invaluable service. This book manages to cover a large amount of material in a unified formalism and to pick out the essential physical ideas and equations for each topic. The book contains brief but adequate accounts of electron lens theory, electron guns, electron detectors, electron interferometry and energy loss spectroscopy, multiple incoherent scattering, dynamical theory for perfect crystals and defects, phase contrast imaging and linear transfer theory, inelastic scattering, crystal structure imaging, analytical microscopy, microdiffraction, and radiation damage.

As with much of the German School, the treatments of electron optics, transfer theory, and interferometry are particularly extensive. A particularly good feature of the book is the incorporation of worthwhile sections on analytical TEM, so that the earlier sections on the physics of multiple incoherent scattering in STEM can be related to the sections on analysis and diffraction contrast. The modern electron microscope must be viewed as a scattering chamber and high-quality electron source fitted with a variety of electron, ion, and photon detectors and spectrometers. This book is the first to even begin to treat the resulting multitude of modes available and physical mechanisms which must be understood on recent analytical TEM/ 